\title{
Branes and the swampland
}

\author{
Hee-Cheol Kim, ${ }^{1}$ Gary Shiu, ${ }^{2}$ and Cumrun Vafa ${ }^{3}$ \\ ${ }^{1}$ Department of Physics, POSTECH, Pohang 790-784, Korea \\ ${ }^{2}$ Department of Physics, 1150 University Avenue, University of Wisconsin, \\ Madison, Wisconsin 53706, USA \\ ${ }^{3}$ Jefferson Physical Laboratory, Harvard University, Cambridge, Massachusetts 02138, USA
}

(Received 10 June 2019; published 5 September 2019)

\begin{abstract}
Completeness of the spectrum of charged branes in a quantum theory of gravity naturally motivates the question of whether the consistency of what lives on the branes can be used to explain some of the swampland conditions. In this paper, we focus on consistency of what lives on string probes to show that some of the theories with $\mathcal{N}=(1,0)$ supersymmetry in ten dimensions and six dimensions, which are otherwise consistent looking, belong to the swampland. Gravitational and gauge group anomaly inflow on these probes can be used to compute the gravitational central charges $\left(c_{L}, c_{R}\right)$ as well as the level of the group's current algebra $k_{L}$. The fact that the left-moving central charge on the string probes should be large enough to allow unitary representations of the current algebra with a given level can be used to rule out some theories. This, in particular, explains why it has not been possible to construct the corresponding theories from string theory.
\end{abstract}

DOI: 10.1103/PhysRevD.100.066006

\section{INTRODUCTION}

Increasing evidence points to the fact that some consistent-looking theories cannot emerge as the IR limit of a quantum gravitational theory and belong to the swampland (see Refs. [1,2] for a recent review for some of the swampland criteria). Ultimately, we would like to explain why the swampland conditions are necessary for the consistency of quantum gravitational theories. There are varying degrees of understanding for different swampland criteria. In this paper, we take a small step to initiate a new direction for a deeper understanding of the swampland criteria: we use the consistency of brane probes to explain why certain consistent-looking supergravity theories coupled to matter that were conjectured not to exist indeed belong to the swampland. See Ref. [3] (also Ref. [4] for a discussion of its generalization) for an early idea of using string and brane probes to constrain type I' string theory.

We focus on $\mathcal{N}=(1,0)$ supergravity theories in ten dimensions and six dimensions (with 16 and 8 supercharges, respectively). These theories enjoy the following common property: the gauge and gravitational anomaly cancellations severely limit the allowed possibilities. In the ten-dimensional case, we are limited to four choices for

Published by the American Physical Society under the terms of the Creative Commons Attribution 4.0 International license. Further distribution of this work must maintain attribution to the author(s) and the published article's title, journal citation, and DOI. Funded by SCOAP. gauge groups [5]: $E_{8} \times E_{8}, S O(32), E_{8} \times U(1)^{248}$, and $U(1)^{496}$. The latter two theories were conjectured to belong to the swampland in Ref. [6]. An argument for this was presented in Ref. [7]. Here, we present an independent argument, ruling out the latter two theories by showing that the left-moving central charge on the BPS (supersymmetric) strings in these theories, which should carry the current algebra of the corresponding group, is too small to realize the latter two theories.

Similarly, anomaly cancellations for six-dimensional $(1,0)$ theories were used to show [8] that there are rather restricted sets of choices for the allowed gauge groups and matter representations. Many of these were realized through $\mathrm{F}$ theory. But it was found that there are infinitely many examples that cancel anomalies but seem not to arise in F theory or any other string realization. These sets arose by having an unbounded rank for the gauge group or an unbounded number of tensors or choices of exotic representations. In this paper, we show that a subset of these theories that could not be realized in $\mathrm{F}$ theory indeed belong to the swampland. In particular, it was shown there [8] that theories with an $S U(N) \times S U(N)$ gauge group with two bifundamental matter representations and additional neutral matter are anomaly free for any $N$. However, only $N \leq 8$ has been realized in string theory. We show that indeed all the theories with $N>9$ belong to the swampland by showing that the central charge of the $S U(N) \times S U(N)$ current algebra on certain BPS strings, which should exist due to the completeness assumption for the spectrum in a gravitational theory $[9,10]$ (see also Ref. [11]), are too 
small to lead to unitary representations for these cases. Moreover, it was found that a family of models with an unbounded number of tensors $T=8 k+9$ and gauge group $\left(E_{8}\right)^{k}$, even though their anomalies cancel, cannot be realized in $\mathrm{F}$ theory except for $k<3$. We show that for a similar reason all these theories are ruled out.

We view this work as just the beginning of the program of using brane probes for a deeper understanding of the swampland conditions. In a first step, we demonstrate the power of this approach with a few examples and with only string probes, but we expect this program to have wider applicabilities in delineating the landscape from the swampland. One can, in principle, consider not just the unitarity of the matter content on the branes but also the consistency between various types of branes and their interactions with one another as other possible ways to better understand the swampland conditions.

The organization of this paper is as follows. In Sec. II, we discuss the consistency conditions of string probes for $\mathcal{N}=(1,0)$ supergravity theories in ten dimensions and show that the two anomaly-free theories with $E_{8} \times U(1)^{248}$ and $U(1)^{496}$ gauge groups are in the swampland. In Sec. III, we discuss similar consistency conditions for sixdimensional theories and show that unitarity of the current algebra on the string probes can be used to rule out several infinite families of anomaly-free six-dimensional $\mathcal{N}=$ $(1,0)$ supergravity theories. We conclude in Sec. IV. Some details are relegated to the Appendixes.

\section{STRINGS IN 10D $\mathcal{N}=(1,0)$ SUPERGRAVITY}

Consistent quantum supergravity theories in ten dimensions are quite limited due to the existence of anomalies. The anomalies of ten-dimensional (10D) $(1,0)$ supergravity theories can be canceled by the Green-Schwarz mechanism [12]. The anomaly cancellation allows only four choices for gauge groups:

$$
S O(32), \quad E_{8} \times E_{8}, \quad E_{8} \times U(1)^{248}, \quad U(1)^{496} .
$$

See Appendix A for details.

The 10D supergravity theories with the former two gauge groups $S O(32)$ and $E_{8} \times E_{8}$ are realized as lowenergy limits of the type I and heterotic string theories. On the other hand, it was argued in Ref. [7] that two other theories with Abelian gauge factors are not consistent at the quantum level due to anomalies in the context of Abelian gauge invariance.

We will now propose a novel stringent condition, ruling out the latter two theories with Abelian gauge factors by using two-dimensional (2D) strings coupled to these 10D theories. When 2D strings couple to the 10D supergravity, the world sheet degrees of freedom (d.o.f.) in general develop local gravitational and gauge anomalies. The world sheet anomalies can be canceled by the anomaly inflow from the $10 \mathrm{D}$ bulk theory toward the $2 \mathrm{D}$ strings. In the following, we will derive the anomaly inflow for 2D strings in the 10D supergravity by employing the method developed in Refs. [13-15]. We will then check if the anomaly inflow can be canceled by local anomalies in a unitary world sheet theory, using the IR properties of the strings and the resulting effective conformal field theory (CFT) on them. When this cancellation cannot occur, the 10D supergravity becomes an inconsistent theory, hosting nontrivial anomalies on the 2D strings.

Strings are sources for the 2-form tensor field $B_{2}$, which by assumption of completeness of the spectrum in a gravitational theory should exist. Moreover, it is easy to show that they are stable due to the BPS condition. A string with tensor charge $Q$ adds to the 10D action the tensor coupling

$$
S^{\mathrm{str}}=Q \int_{\mathcal{M}_{10}} B_{2} \wedge \prod_{a=1}^{8} \delta\left(x^{a}\right) d x^{a}=Q \int_{\mathcal{M}_{2}} B .
$$

The 2-form $B$ transforms under the local gauge and the local Lorentz symmetry $[16,17]$ (with parameters $\Lambda_{i}$ and $\Theta$, respectively) as

$$
B_{2} \rightarrow B_{2}-\frac{1}{4} \sum_{i} \operatorname{Tr}\left(\Lambda_{i} F_{i}\right)+\operatorname{tr}(\Theta R),
$$

where $F_{i}$ denotes the gauge field strengths and $R$ denotes the curvature 2-form of the 10D spacetime.

The string action $S^{\text {str }}$ is not invariant under these local transformations,

$$
\delta_{\Lambda, \Theta} S^{\mathrm{str}}=Q \int_{\mathcal{M}_{2}}\left[-\frac{1}{4} \sum_{i} \operatorname{Tr}\left(\Lambda_{i} F_{i}\right)+\operatorname{tr}(\Theta R)\right] .
$$

As a consequence, the introduction of 2D strings induces an anomaly inflow along the world sheet of the strings. The anomaly inflow is characterized by the 4-form anomaly polynomial, which in this case is given by

$$
I_{4}^{\text {inflow }}=Q\left(-\frac{1}{4} \sum_{i} \operatorname{Tr} F_{i}^{2}+\operatorname{tr} R^{2}\right)
$$

These anomalies must be canceled by the anomalies coming from the world sheet d.o.f. living on the strings.

A half-BPS string coupled to the 10D supergravity gives rise to an $\mathcal{N}=(0,8)$ superconformal field theory (SCFT) at low energy. To find the chirality of the supersymmetry, one uses the condition that we start with a chiral theory in ten dimensions, and for a BPS string, we preserve half of the supersymmetries, leading to a definite chirality for the supercurrents on the world sheet. Supersymmetry on the BPS string also shows that the current for the group has chirality opposite that of supersymmetry. We choose 
conventions so that the supersymmetry current is right moving and the current for the group is left moving.

To cancel the anomaly inflow from the bulk gravity theory, the gravitational and the gauge anomalies of the SCFT on a string must be

$$
\begin{aligned}
I_{4} & =-I_{4}^{\text {inflow }} \\
& =Q\left[\frac{1}{2} p_{1}\left(T_{2}\right)-c_{2}(S O(8))+\frac{1}{4} \sum_{i} \operatorname{Tr} F_{i}^{2}\right] .
\end{aligned}
$$

Here, we used the decomposition

$$
\operatorname{tr} R^{2}=-\frac{1}{2} p_{1}\left(T_{2}\right)+c_{2}(S O(8)),
$$

where $p_{1}\left(T_{2}\right)$ is the first Pontryagin class of the 2-manifold $\mathcal{M}_{2}$ and $c_{2}(S O(8))$ is the second Chern class of the $S O(8)$ R-symmetry bundle of the world sheet theory.

Note that the above result involves the contribution from the center-of-mass d.o.f. The center-of-mass modes form a free $(0,8)$ multiplet $\left(X_{\mu}, \lambda_{+}^{I}\right)$ with $\mu, I=1, \ldots, 8$, where $X_{\mu}$ parametrize the motion of strings along eight transverse directions and $\lambda_{+}^{I}$ is the right-moving fermion in the $S O(8)$ spinor representation. From this, we read the anomaly polynomial for the center-of-mass modes:

$$
I_{4}^{\mathrm{com}}=-\frac{1}{6} p_{1}\left(T_{2}\right)-c_{2}(S O(8)) .
$$

So, the anomaly polynomial of the interacting sector in the $2 \mathrm{D}$ world sheet SCFT is given by $I_{4}^{\prime}=I_{4}-I_{4}^{\mathrm{com}}$.

Let us now focus on the 2D SCFT on a single string, i.e., $Q=1$. The anomaly polynomial of this CFT is

$$
I_{4}^{\prime}=I_{4}-I_{4}^{\mathrm{com}}=\frac{2}{3} p_{1}\left(T_{2}\right)+\frac{1}{4} \sum_{i} \operatorname{Tr} F_{i}^{2}
$$

The left-moving and the right-moving central charges $c_{L}$ and $c_{R}$ and the level $k_{i}$ 's of gauge algebras in the world sheet SCFT can be computed from the anomaly polynomial $I_{4}^{\prime}$. The relative central charge $c_{R}-c_{L}$ is the coefficient of the gravitational anomaly $-\frac{1}{24} p_{1}\left(T_{2}\right)$, and the rightmoving central charge is $c_{R}=3 k_{R}$, where $k_{R}$ is the 't Hooft anomaly coefficient of the superconformal R-symmetry current at the IR fixed point. One finds that 't Hooft anomalies for the $S O(8) \mathrm{R}$ symmetry in $I_{4}^{\prime}$ vanish. The level $k_{i}$ is the coefficient of the gauge anomaly term $\frac{1}{4} \operatorname{Tr} F_{i}^{2}$. We then compute

$$
c_{L}=16, \quad c_{R}=0, \quad k_{i}=1 .
$$

The central charges are constrained by unitarity conditions on 2D CFTs, which can be viewed as IR d.o.f. on the strings. The central charge realizing the level- $k$ Kac-Moody algebra of group $G$ is (see, e.g., Ref. [18])

$$
c_{G}=\frac{k \cdot \operatorname{dim} G}{k+h^{\vee}}
$$

where $\operatorname{dim} G$ is the dimension and $h^{\vee}$ is the dual Coxeter number of group $G$, respectively. The central charge for $U(1)$ current algebra is $c_{U(1)}=1$ for any $k_{U(1)}$. For $(0,8)$ SCFTs, the current algebra for group $G$ is on the leftmoving sector. This tells us that

$$
\sum_{i} c_{i}=\sum_{i} \frac{k_{i} \cdot \operatorname{dim} G_{i}}{k_{i}+h_{i}^{\vee}} \leq c_{L}
$$

for a unitary CFT on a string.

We find that the 10D supergravity theories with Abelian gauge groups contains nonunitary strings violating this inequality. The $U(1)^{496}$ and $U(1)^{248}$ Abelian factors in these theories give rise to too many left-moving modes for the current algebras in the world sheet CFT, and the central charge of the current algebra exceeds $c_{L}=16$, namely, $\sum_{i} c_{i}>c_{L}$. Therefore, we conclude that 10D supergravity theories with $U(1)^{496}$ and $E_{8} \times U(1)^{248}$ gauge groups are inconsistent when coupled to 2D strings, and thus they belong to the swampland. On the other hand, the central charges on a single string in the 10D supergravities with an $S O(32)$ or $E_{8} \times E_{8}$ gauge group saturate the bound (12) as $\sum_{i} c_{i}=c_{L}=16$, so the string can consistently couple to these $10 \mathrm{D}$ theories.

\section{STRINGS IN 6D $\mathcal{N}=(1,0)$ SUPERGRAVITY}

We now turn to six-dimensional (6D) supergravity theories preserving eight supersymmetries. There are four kinds of massless supermultiplets appearing in such theories: a gravity multiplet, tensor multiplets, vector multiplets, and hypermultiplets. Six-dimensional supergravity theories may have anomalies, which are characterized by an 8-form anomaly polynomial $I_{8}$, from the chiral fields in these multiplets.

Let us consider a gravity theory coupled to $T$ tensor multiplets and vector multiplets of the gauge group $G=\prod_{i} G_{i}$, as well as hypermultiplets transforming in representation $\mathbf{R}$ of the gauge group. The chiral fields such as the self-dual and anti-self dual two-forms $B_{\mu \nu}^{ \pm}$, a gravitino, and other chiral fermions in this theory contribute to the anomalies for the gauge and Lorentz transformations. Such anomalies can exactly be computed by evaluating one-loop box diagrams for the chiral fields with four external gravitational and gauge sources. Consistent quantum supergravity theories must be free of such anomalies. Thus, nonvanishing one-loop anomalies must be canceled for the $6 \mathrm{D}$ theories that are consistent at the quantum level, which leads to quite stringent constraints.

The one-loop anomalies can be canceled by the GreenSchwarz-Sagnotti mechanism [19] if the anomaly polynomial factorizes as 
TABLE I. Group theory factors.

\begin{tabular}{ccccccccc}
\hline \hline$G$ & $S U(N)$ & $S O(N)$ & $S p(N)$ & $G_{2}$ & $F_{4}$ & $E_{6}$ & $E_{7}$ & $E_{8}$ \\
\hline$\lambda$ & 1 & 2 & 1 & 2 & 6 & 6 & 12 & 60 \\
\hline \hline
\end{tabular}

$$
\begin{aligned}
I_{8}^{1-\text { loop }} & =\frac{1}{2} \Omega_{\alpha \beta} X_{4}^{\alpha} X_{4}^{\beta}, \\
X_{4}^{\alpha} & =\frac{1}{2} a^{\alpha} \operatorname{tr} R^{2}+\frac{1}{4} \sum_{i} b_{i}^{\alpha} \frac{2}{\lambda_{i}} \operatorname{tr} F_{i}^{2},
\end{aligned}
$$

where $\Omega_{\alpha \beta}$ is a symmetric bilinear form of $T+1$ tensors with a signature $(1, T)$ and $a^{\alpha}$ and $b_{i}^{\alpha}$ are vectors in $\mathbb{R}^{1, T}$ and $\lambda_{i}$ is a group theory factor given in Table I.

The conditions for the factorization can be summarized as

$$
\begin{aligned}
H-V & =273-29 T, \quad a \cdot a=9-T, \\
0 & =B_{\mathbf{a d j}}^{i}-\sum_{\mathbf{R}} n_{\mathbf{R}}^{i} B_{\mathbf{R}}^{i}, \\
a \cdot b_{i} & =\frac{\lambda_{i}}{6}\left(A_{\mathbf{a d j}}^{i}-\sum_{\mathbf{R}} n_{\mathbf{R}}^{i} A_{\mathbf{R}}^{i}\right), \\
b_{i} \cdot b_{i} & =\frac{\lambda_{i}^{2}}{3}\left(\sum_{\mathbf{R}} n_{\mathbf{R}}^{i} C_{R}^{i}-C_{\mathbf{a d j}}^{i}\right), \\
b_{i} \cdot b_{j} & =2 \lambda_{i} \lambda_{j} \sum_{\mathbf{a d j}} n_{\mathbf{a d j}}^{i j} A_{\mathbf{R}}^{i} A_{\mathbf{S}}^{j} \quad(i \neq j),
\end{aligned}
$$

where $\Omega_{\alpha \beta}$ is used for the inner product of two vectors, like $v \cdot w=\Omega_{\alpha \beta} v^{\alpha} w^{\beta}$. Here, $V$ and $H$ are the number of vector and hyper multiplets; $n_{\mathbf{R}}^{i}$ denotes the number of hypermultiplets in the representation $\mathbf{R}$ for gauge group $G_{i}$; and $A_{\mathbf{R}}^{i}, B_{\mathbf{R}}^{i}$, and $C_{\mathbf{R}}^{i}$ are group-theory factors for each representation defined as follows:

$\operatorname{tr}_{\mathbf{R}} F^{2}=A_{\mathbf{R}} \operatorname{tr} F^{2}, \quad \operatorname{tr}_{\mathbf{R}} F^{4}=B_{\mathbf{R}} \operatorname{tr} F^{4}+C_{\mathbf{R}}\left(\operatorname{tr} F^{2}\right)^{2}$.

When these conditions are satisfied, the perturbative anomaly factorizes, and it can be canceled by adding to the action the Green-Schwarz term

$$
S_{\mathrm{GS}}=\int \Omega_{\alpha \beta} B_{2}^{\alpha} \wedge X_{4}^{\beta}
$$

This term induces tree-level anomalies of the form $I_{8}^{\mathrm{GS}}=$ $-\frac{1}{2} \Omega_{\alpha \beta} X_{4}^{\alpha} X_{4}^{\beta}$ that exactly cancels the factorized anomaly $I_{8}^{1-\text { loop }}$. So, $6 \mathrm{D}$ supergravity theories satisfying the conditions in Eq. (14) have no apparent quantum anomalies and seem to be consistent. Extensive lists of would-be consistent $6 \mathrm{D}$ supergravity theories are given in various literature [8,20-27] (see Ref. [4] for a review).

\section{A. Central charges of 2D $(0,4)$ SCFTs on strings}

Let us now consider 2D strings in 6D supergravity theory without manifest anomalies. We will discuss additional conditions from the $6 \mathrm{D} / 2 \mathrm{D}$ coupled system. Strings are sources for the 2 -form fields $B_{2}^{\alpha}$ and thus should exist by the assumption of completeness of the spectrum in a gravitational theory. We shall consider BPS strings preserving half of the supersymmetries. The world sheet theory on those strings gives rise to $2 \mathrm{D}(0,4)$ SCFT at low energy. As discussed in the 10D cases, the d.o.f. living on the string world sheet can have nonzero anomalies, and these anomalies must be canceled through the anomaly inflow mechanism. The anomaly inflow in 6D SCFTs was studied in Refs. [28,29] (see also Ref. [30] for generalization to 6D supergravities from F-theory compactification). See Appendix B for a brief review on the anomaly inflow to 2D strings in 6D SCFTs and 6D supergravity theories.

The 2D SCFT on strings with charge $Q^{\alpha}$ in the 6D supergravity theory has the anomaly polynomial of this form:

$$
\begin{aligned}
I_{4}= & \Omega_{\alpha \beta} Q^{\alpha}\left(\frac{1}{2} a^{\alpha} \operatorname{tr} R^{2}+\frac{1}{4} \sum_{i} b_{i}^{\alpha} \operatorname{Tr} F_{i}^{2}+\frac{1}{2} Q^{\beta} \chi_{4}\left(N_{4}\right)\right) \\
= & -\frac{Q \cdot a}{4} p_{1}\left(T_{2}\right)+\frac{1}{4} \sum_{i} Q \cdot b_{i} \operatorname{Tr} F_{i}^{2} \\
& -\frac{Q \cdot Q-Q \cdot a}{2} c_{2}(R)+\frac{Q \cdot Q+Q \cdot a}{2} c_{2}(l) .
\end{aligned}
$$

In this computation, we used the decomposition $\operatorname{tr} R^{2}=$ $-\frac{1}{2} p_{1}\left(T_{2}\right)+c_{2}(l)+c_{2}(R)$.

This result involves the contribution from the center-ofmass d.o.f., which decouples in the IR SCFT. The centerof-mass modes consist of four bosons common to left and right movers and four right-moving fermions, and they form a free hypermultiplet $\left(X_{a \dot{a}}, \lambda_{a}\right)$, where $a$ and $\dot{a}$ are indices for $S U(2)_{l} \times S U(2)_{R}$. They contribute to the anomaly as

$$
I_{4}^{\mathrm{com}}=-\frac{1}{12} p_{1}\left(T_{2}\right)-c_{2}(l)
$$

Therefore, the anomaly polynomial of the 2D world sheet theory after removing the center-of-mass contributions becomes

$$
\begin{aligned}
I_{4}^{\prime}= & I_{4}-I_{4}^{\mathrm{com}} \\
= & -\frac{3 Q \cdot a-1}{12} p_{1}\left(T_{2}\right)+\frac{1}{4} \sum_{i} Q \cdot b_{i} \operatorname{Tr} F_{i}^{2} \\
& -\frac{Q \cdot Q-Q \cdot a}{2} c_{2}(R)+\frac{Q \cdot Q+Q \cdot a+2}{2} c_{2}(l) .
\end{aligned}
$$

The central charges of the 2D SCFT can be extracted from the anomaly polynomial as discussed in the previous 
section. The relative central charge $c_{R}-c_{L}$ is again the coefficient of the gravitational anomaly. The right-moving central charge $c_{R}$ is associated to the anomaly coefficient of the R-symmetry current. Here, we should be careful about the R symmetry at the IR fixed point. It is possible that an accidental symmetry emerges at low energy and it takes over the role of the R symmetry in the IR $(0,4)$ superconformal algebra. It is also possible that a $2 \mathrm{D}$ world sheet theory degenerates to a product of distinct SCFTs carrying different IR R symmetries.

Indeed, this happens for the strings in local 6D SCFTs or little string theories (LSTs) embedded in the supergravity theories. The 2D SCFTs on such strings have an accidental $S U(2)_{I}$ symmetry in the decoupling limit, and this symmetry becomes the $S U(2)$ R symmetry in the $(0,4)$ superconformal algebra. This $S U(2)_{I}$ is descended from the $S U(2)$ R symmetry of the local 6D SCFTs or LSTs, but it is broken in the full supergravity theory. The free theory with the center-of-mass d.o.f. we discussed above also has the same accidental $S U(2)_{I}$ symmetry.

It is therefore crucial to identify the right R symmetry in the IR SCFTs. Only after this, we can extract the correct central charges in the IR SCFTs. From now on, we will focus on the strings in the 6D supergravity theory that give rise to a single interacting SCFT at low energy without the accidental $S U(2)_{I}$ symmetry. The IR SCFTs on such supergravity strings (not strings in local 6D SCFTs or LSTs) have the $(0,4)$ superconformal algebra with an $S U(2)_{R}$ R symmetry. The conditions for this type of strings will be given below. The right-moving central charge $c_{R}$ of these SCFTs can then be read off from the anomaly coefficient of the $S U(2)_{R}$ symmetry. For a nondegenerate 2D SCFT on a supergravity string, the central charges $c_{L}$ and $c_{R}$ are given by

$$
c_{L}=3 Q \cdot Q-9 Q \cdot a+2, \quad c_{R}=3 Q \cdot Q-3 Q \cdot a .
$$

The central charges $k_{i}$ and $k_{l}$ for the bulk gauge symmetries $G_{i}$ and $S U(2)_{l}$ can also be extracted from the anomaly polynomial. We find

$$
k_{i}=Q \cdot b_{i}, \quad k_{l}=\frac{1}{2}(Q \cdot Q+Q \cdot a+2) .
$$

A large class of $6 \mathrm{D}(1,0)$ supergravity theories can be engineered in $\mathrm{F}$ theory on elliptic Calabi-Yau 3-folds. In the context of F theory, the 2D SCFT with string charge $Q$ arises as a low-energy theory on a D3-brane wrapping genus $g$ curve $C=Q$ in the base $B$ of the 3 -fold. We can compare the above results against the central charges of the strings coming from D3-branes in F theory. The 2D SCFT for a D3-brane wrapping a genus $g$ curve $C$ inside $B$ has the central charges [31] (see also Ref. [30])

$c_{L}^{\prime}=3 C \cdot C-9 K \cdot C+6, \quad c_{R}^{\prime}=3 C \cdot C-3 K \cdot C+6$, where $K$ is the canonical class of $B$, and it has an $S U(2)_{l}$ current algebra at level $k_{l}^{\prime}=g-1$. Here, the genus $g$ of the curve $C$ can be computed by the Riemann-Roch theorem

$$
C \cdot C+K \cdot C=2 g-2 .
$$

These results again include the contribution from the center-of-mass modes; four left-moving and four rightmoving bosons and four right-moving fermions. The central charges of the center-of-mass modes are $c_{L}^{\text {com }}=4$ and $c_{R}^{\text {com }}=6$, and as discussed in Ref. [31], they contribute to the $S U(2)_{l}$ current algebra by $k_{l}^{\text {com }}=-1$.

One can easily see that the central charges $c_{L}^{\prime}, c_{R}^{\prime}$, and $k_{l}^{\prime}$ in F-theory models after removing the center-of-mass contributions are in perfect agreement with the central charges of 2D SCFTs from the anomaly inflow given in (20) and (21). To see this agreement, one needs to identify the inner product $\Omega$ among tensors with the intersection form in $H_{2}(B, \mathbb{Z})$ and map the vector $a$ to the canonical class $K$ in the base of the elliptic $\mathrm{CY}_{3}$. This comparison confirms our anomaly inflow computation for $2 \mathrm{D}$ strings in $6 \mathrm{D}$ supergravity theories.

\section{B. Consistency conditions}

We shall now show that the consistency of 2D world sheet theories encoded in the central charges imposes additional conditions on 6D supergravity theories.

Let us consider the moduli space of a 6D supergravity theory that is parametrized by scalar fields in the tensor multiplets as well as the scalar field in the hypermultiplet controlling the overall volume of the tensor moduli space. From supergravity considerations, for this moduli space being well defined, we should be able to find a linear combination of these scalar fields, which we call $J$, satisfying

$$
J \cdot J>0, \quad J \cdot b_{i}>0, \quad-J \cdot a>0 .
$$

This $J$ plays the role of the central charge in the supersymmetry algebra for the $B$ fields. The first condition stands for the metric positivity of the tensor branch along $J$. The second one is the condition for the gauge kinetic term along $J$ to have proper sign on the tensor moduli [19]. Otherwise, the gauge kinetic term has a wrong sign, and it leads to an instability. The last condition ensures, through supersymmetry, the positivity of the Gauss-Bonnet term in gravity [32]. While there have been attempts to prove the positivity of the curvature-squared corrections in $D>4$ using, e.g., unitarity [33], the singular UV behavior due to graviton exchange prevents one from making such a spectral decomposition argument [34]. Here, we note that, even if we impose this last condition, there seem to be infinitely many anomaly-free 6D supergravity theories (see Ref. [4] for a review). We thus assume its validity, leaving a derivation for future work. 
In F-theory realization [35], this combination $J$ corresponds to a Kähler form $J \in H^{1,1}(B)$ of the base $B$. The above conditions on $J$ define a positive-definite Kähler cone on $B$. We will call $J$ a Kähler form for all $6 \mathrm{D}$ theories, regardless of whether it has an F-theory realization.

The tensions of 2D BPS strings are determined with respect to the Kähler form $J$. This imposes a condition $Q \cdot J \geq 0$ on the string charge $Q$. A world sheet theory has non-negative tension only if $Q \cdot J \geq 0$.

The strings with $Q \cdot J \geq 0$ embedded in 6D supergravity theories must give rise to unitary 2D SCFTs. For a unitary 2D CFT, the central charges must be non-negative, i.e., $c_{L}$, $c_{R} \geq 0$. If the central charges computed through the anomaly inflow for a string are negative, the corresponding anomalies cannot be canceled by a unitary $2 \mathrm{D}$ world sheet theory. This results in the 6D supergravity theory with such strings being inconsistent, hosting nonvanishing anomalies along the 2D string world sheet, and it thus belongs to the swampland. So, we can use the anomaly inflow on 2D strings to analyze the consistency of $6 \mathrm{D}$ supergravity theories.

We remark that the strings in 6D SCFTs or LSTs contained in $6 \mathrm{D}$ supergravity theories in general lead to 2D CFTs having a negative value for $c_{R}$ given in (20). For example, the unit string charge $Q$ for a $2 \mathrm{D}$ string in the 6D $S O(8)$ non-Higgsable SCFT have the properties $Q \cdot Q=-4$ and $Q \cdot K=+2$. So, the value for $c_{R}$ of this string with unit charge $Q$ is -18 . This seems to say that the theory is inconsistent since its central charge is negative $c_{R}<0$ by the formula in (20). However, this is not the case. Note that the central charge $c_{R}$ above is obtained by assuming the R symmetry of the low-energy $(0,4)$ SCFT is the $S U(2)_{R}$. As discussed, the strings in local 6D SCFTs or LSTs have an accidental $S U(2)_{I}$ symmetry, and this becomes the R symmetry of the low-energy SCFT. Therefore, $c_{R}$ in such strings is different from what we computed above. The central charges of various world sheet theories in 6D SCFTs are computed in the literature $[28,29]$, and one can check that those theories have positive central charges $c_{R}$ and $c_{L}$ with respect to the $S U(2)_{I} \mathrm{R}$ symmetry.

We are interested in the configurations of a single string in the $6 \mathrm{D}$ supergravity that have $S U(2)_{R}$ as the R symmetry in the superconformal algebra and that do not degenerate to a product of disconnected 2D SCFTs at low energy. A single string state has no bosonic zero mode along the transverse $\mathbb{R}^{4}$ directions except the center-of-mass d.o.f. This implies that, after removing the center of mass modes, the world sheet theory on a string contains the $S U(2)_{l}$ current algebra realized on the left movers. So, the $S U(2)_{l}$ central charges should be non-negative, i.e., $k_{l} \geq 0$. In F-theory compactification, this condition becomes a trivial condition, saying that $g \geq 0$ for a string wrapped on a genus $g$ curve $Q$. The central charge conditions $c_{R} \geq 0$ and $k_{l} \geq 0$ on these SCFTs can be summarized as

$$
Q \cdot Q \geq-1, \quad Q \cdot Q+Q \cdot a \geq-2 .
$$

There are more conditions associated to the flavor central charges $k_{i}=Q \cdot b_{i}$. The flavor central charge measures the index of the bulk fields charged under the gauge group $G_{i}$ on the string background with charge $Q$. So, it counts the number of zero modes at the intersection between the tensor carrying the gauge group $G_{i}$ and the tensor labeled by the string charge $Q$. Unless the string degenerates to an instanton string of the group $G_{i}$, namely, unless $Q \sim b_{i}$, the flavor central charge can receive contributions only from fermionic zero modes that are in the left-moving sector. This means that the flavor central charges of the 2D SCFTs on nondegenerate strings (not in local 6D SCFTs or LSTs) in $6 \mathrm{D}$ supergravities should be non-negative. In other words, for the strings we are interested in,

$$
k_{i}=Q \cdot b_{i} \geq 0
$$

where we used the convention that left movers have positive contributions to flavor central charges. In the F-theory viewpoint, the condition (26) is the same as the condition that the curve class $Q$ is effective and irreducible within the Mori cone of the Kähler base $B$.

Note that a 2D theory on instanton strings can have right movers associated to bosonic zero modes parametrizing the moduli space of $G_{i}$ instantons. These right movers can provide negative contributions to the flavor central charges. However, such instanton strings correspond to the strings in local 6D SCFTs or 6D LSTs. When a string degenerates to a product of the instanton strings, the low-energy theory will include 2D theories for the strings in local 6D SCFTs or LSTs that have the accidental $S U(2)_{I} \mathrm{R}$ symmetry. As discussed above, we are not interested in the world sheet theories with $S U(2)_{I}$ R symmetry. So, we shall only focus on strings and the associated 2D SCFTs satisfying the condition (26) as well as (25).

We claim that the conditions (25) and (26) as well as $c_{L}>0$ are also sufficient conditions for the associated string being a nondegenerate string in the gravity theory. Consider compactification of the 6D gravity theory on a circle and also a string wrapped around the circle with momentum $p=n / R$ along the circle. For large $n$, the string forms a black hole in the five-dimensional (5D) supergravity, and the black hole entropy goes as $S \sim \sqrt{c_{L} n}$ [31,36,37]. The conditions (25), (26), and $c_{L}>0$ are precisely the conditions for the string forming a $5 \mathrm{D}$ black hole with nontrivial entropy, and therefore the string must be a nondegenerate string in the gravity theory. Note that such a 5D black hole cannot descend from a string in the 6D CFTs or LSTs upon a circle compactification.

For 2D SCFTs on nondegenerate strings in supergravity theories, we have $G_{i}$ [including $S U(2)_{l}$ ] current algebra with level $k_{i}$. Using supersymmetry algebra in the context of BPS strings, one can show that the current algebra is on 
the left movers in the $(0,4)$ SCFTs, and its central charge contribution is given in (11). Therefore, we find the following constraint on the 2D world sheet SCFT in the 6D supergravity:

$$
\sum_{i} \frac{k_{i} \cdot \operatorname{dim} G_{i}}{k_{i}+h_{i}^{\vee}} \leq c_{L} .
$$

So, the 2D SCFTs on strings satisfying the conditions in Eqs. (25) and (26) must have central charges constrained by Eq. (27). Otherwise, the 2D world sheet theory is nonunitary. In conclusion, we claim that a $6 \mathrm{D}$ supergravity theory embedding 2D strings of which the world sheet theory violates the condition (27) is inconsistent and it therefore belongs to the swampland.

\section{Examples}

The basic structure of our examples is as follows. For each one, we have the $\Omega, a$, and $b_{i}$ given by anomaly cancellation conditions. We use this to find the allowed ranges for $J$ and choose a particular $J$ in the allowed region. We then use this to restrict the allowed string charges $Q$ 's and use that to compute central charges $c_{R}$ and $c_{L}$ and $k_{l}$ and $k_{i}$ and see if we have any contradictions with unitarity.

Let us first consider the 6D supergravity theory coupled to $T=9$ tensors with the $S U(N) \times S U(N)$ gauge group and two bifundamental hypermultiplets introduced in Ref. [8] (see also Ref. [20] for $T=1$ models). The anomaly polynomial of this model factorizes for an arbitrary $N$, and hence it seems that they provide an infinite family of consistent 6D supergravity theories. It was, however, shown in Ref. [8] that these models have no F-theory realization at large enough $N$.

Let us examine these models with 2D strings to see if the consistency conditions of the world sheet theory on the strings can provide any bound on $N$.

We can always choose a tensor basis such that the bilinear form $\Omega$ and the vectors $a, b_{1}$, and $b_{2}$ are given as follows [8]:

$$
\begin{aligned}
& \Omega=\operatorname{diag}\left(+1,(-1)^{9}\right), \quad a=\left(-3,(+1)^{9}\right), \\
& b_{1}=\left(1,-1,-1,-1,0^{6}\right), \quad b_{2}=\left(2,0,0,0,(-1)^{6}\right) .
\end{aligned}
$$

In this basis, one can easily see that a Kähler form chosen as $J=\left(1,0^{9}\right)$ satisfies the conditions $J^{2}>0, J \cdot b>0$, and $J \cdot a<0$.

Consider a string of a generic charge $Q=\left(q_{0}, q_{1}\right.$, $\left.\ldots, q_{9}\right)$ with $q_{i} \in \mathbb{Z}$. This string with $q_{0}>0$ has a positive tension with respect to $J$. The conditions (25) and (26) on the IR SCFT for this string can be summarized as

$$
\begin{aligned}
q_{0}^{2}-\sum_{i=1}^{9} q_{i}^{2} & \geq-1, \quad q_{0}^{2}-\sum_{i=1}^{9} q_{i}^{2}-3 q_{0}-q_{1: 3}-q_{4: 9} \geq-2, \\
k_{1} & =q_{0}+q_{1: 3} \geq 0, \quad k_{2}=2 q_{0}+q_{4: 9} \geq 0,
\end{aligned}
$$

where $q_{1: 3} \equiv \sum_{i=1}^{3} q_{i}$ and $q_{4: 9} \equiv \sum_{i=4}^{9} q_{i}$. In addition, the flavor central charges are restricted by the unitarity bound (27)

$$
\frac{k_{1}\left(N^{2}-1\right)}{k_{1}+N}+\frac{k_{2}\left(N^{2}-1\right)}{k_{2}+N} \leq c_{L},
$$

where the left-moving central charge is

$c_{L}=3\left(q_{0}^{2}-\sum_{i=1}^{9} q_{i}^{2}\right)+9\left(3 q_{0}+q_{1: 3}+q_{4: 9}\right)+2$.

As discussed above, if this bound is violated for any $Q$ satisfying (29), the anomaly inflow from the bulk 6D supergravity theory cannot be canceled by a unitary $2 \mathrm{D}$ CFT which renders the 6D supergravity inconsistent at the quantum level.

The bound (30) gives the strongest constraint on $N$ of the $6 \mathrm{D}$ supergravity theory when the left-hand side is maximized, namely, $k_{i}$ 's are minimized, while the right-hand side is minimized. This implies the strongest bound can be given by a string with $q_{0}^{2}-\sum_{i} q_{i}^{2}=-1$ and $k_{1}=0$, $k_{2}=1$. This occurs for $Q=\left(1,-1,0,0,-1,0^{5}\right)$. The central charge bound for the string configuration being unitary is

$$
\frac{k_{2}\left(N^{2}-1\right)}{k_{2}+N} \leq c_{L} \rightarrow \frac{N^{2}-1}{1+N} \leq 8 \rightarrow N \leq 9 .
$$

Therefore, the 6D supergravity theory with $N>9$ belongs to the swampland containing nonunitary string configurations. This bound is stronger than the bound $N \leq 12$ from the Kodaira condition in F theory [8]. It is interesting that we can thus rule out would-be purely geometric constructions that could have in principle realized this model for $N=10,11,12$. In other words, our arguments can be used to teach us some facts about the geometry of elliptic CalabiYau 3-folds. Also, it is reassuring that this bound does not rule out the string theory realization for $N=8$ given in Refs. [38,39] and all the $N \leq 8$ theories which one can obtain from it by partial Higgsing. Remarkably, our world sheet analysis provides a new bound on the rank of gauge groups in the $6 \mathrm{D}$ bulk supergravity theory, and the result is consistent with the F-theory argument and also the known string theory realization. It would be interesting to see if one can construct the $N=9$ case, which we were not able to rule out.

The second example is the 6D supergravity with $T=1$ and the $S U(N)$ gauge group coupled to one symmetric and $N-8$ fundamental hypermultiplets first introduced in 
Refs. [8,40]. The rank of the gauge group is bounded as $N \leq 30$ from the 6D anomaly cancellation conditions. For this model, we are free to choose a tensor basis giving

$\Omega=\operatorname{diag}(1,-1), \quad a=(-3,1), \quad b=(0,-1)$.

The Kähler form can always be chosen as $J=(n, 1)$ with $n^{2}>1$ and $n>0$. This theory has no F-theory realization because when we identify the base $B$ with a Hirzebruch surface $\mathbb{F}_{1}$ the tensor for $b$ cannot be mapped to any effective curve class [8].

We shall now see if the consistency conditions on string configurations of this $6 \mathrm{D}$ theory can provide a stronger bound on the rank $N$. Consider a generic string with $Q=$ $\left(q_{1}, q_{2}\right)$ satisfying the conditions (25) and (26), namely,

$$
\begin{aligned}
q_{1}^{2}-q_{2}^{2} & \geq-1, \quad q_{1}^{2}-q_{2}^{2}-3 q_{1}-q_{2} \geq-2, \\
k & =Q \cdot b=q_{2} \geq 0 .
\end{aligned}
$$

Also, $n q_{1}>q_{2}$ from $J \cdot Q>0$. These conditions can be then simplified, for the strings interacting with the gauge group, as

$$
q_{1} \geq 3 \quad q_{1}-2 \geq q_{2}>0 .
$$

The constraint on the central charges

$$
\frac{q_{2}\left(N^{2}-1\right)}{q_{2}+N} \leq 3\left(q_{1}^{2}-q_{2}^{2}\right)+9\left(3 q_{1}+q_{2}\right)+2
$$

can provide the strongest bound on $N$ when $Q=(3,1)$, and the bound is $N \leq 117$. This bound is weaker than the bound $N \leq 30$ coming from the $6 \mathrm{D}$ anomaly cancellation conditions. This may imply, unless another inconsistency is revealed by any other means, that these 6D supergravity models with $N \leq 30$ are all consistent theories, though they do not seem to admit an F-theory realization.

The anomaly inflow consideration can provide a new bound on a family of models with $T=8 k+9$ and gauge group $G=\left(E_{8}\right)^{k}$ for arbitrary large $k$, which was introduced in Ref. [8]. The vectors $a$ and $b_{i}$ in the anomaly polynomial satisfy $a \cdot b_{i}=10, b_{i} \cdot b_{j}=-2 \delta_{i j}$ with $i$, $j=1, \ldots, k$. When $k \geq 3$, one can choose a basis for tensors in Ref. [8] that gives rise to

$$
\begin{aligned}
& \Omega=\operatorname{diag}\left(1,(-1)^{8 k+9}\right), \quad a=\left(-3,1^{8 k+9}\right), \\
& b_{i}=\left(-1,-1,0^{4(i-1)},(-1)^{3},-3,0^{8 k+8-4 i}\right),
\end{aligned}
$$

The Kähler form in this basis can be chosen as

$J=\left(-j_{0}, 0^{4 k+1}, 1^{4 k+8}\right), \quad(4 k+8) / 3>j_{0}>\sqrt{4 k+8}$.

Now, consider a string with charge $Q=\left(-q, 0^{8 k+9}\right)$ in this 6D model. This string has a positive tension if $q>0$.
Moreover, the conditions $k_{l} \geq 0, c_{R} \geq 0$ and $k_{i} \geq 0$ can be satisfied if $q>2$. However, the bound on the levels of flavor current algebras $k_{i}=Q \cdot b_{i}=q$,

$$
\sum_{i=1}^{k} \frac{248 k_{i}}{k_{i}+30} \leq c_{L} \rightarrow k \frac{248 q}{q+30} \leq 3 q(q-9)+2,
$$

cannot be satisfied by, for example, strings with charge $3 \leq q \leq 14$ for any $k \geq 3$. This result demonstrates that all these 6D supergravity models for $k \geq 3$ endowed with the bilinear form $\Omega$ and vectors $a$ and $b_{i}$ given in (37) reveal nonvanishing anomalies on the $2 \mathrm{D}$ strings, and therefore they are in the swampland.

Note, however, that the 6D supergravity theories of this type for $k \leq 2$ are not ruled out by this analysis. When $k=1,2$, there exists other solutions of $\Omega$ and $a, b_{i}$ canceling the anomalies, like

$$
\begin{aligned}
& \Omega=\operatorname{diag}\left(1,(-1)^{17}\right), \quad a=\left(-3,1^{17}\right), \\
& b_{1}=\left(0,1,(-1)^{11}, 0^{5}\right)
\end{aligned}
$$

for $k=1$ and

$$
\begin{aligned}
& \Omega=\operatorname{diag}\left(1,(-1)^{25}\right), \quad a=\left(-3,1^{25}\right), \\
& b_{1}=\left(0,1,(-1)^{11}, 0^{13}\right), \quad b_{2}=\left(0,0^{13}, 1,(-1)^{11}\right)
\end{aligned}
$$

for $k=2$. Thus, the above analysis does not apply to the $k=1,2$ cases. We do not find any string configuration showing inconsistencies for these cases. Indeed, the 6D gravity theory with $k=2$ can be realized by the compactification of M theory on $K 3 \times\left(S^{1} / \mathbb{Z}_{2}\right)$, where we place 24 M5 branes on the interval [41].

The last example is the $6 \mathrm{D}$ supergravity theory with $T=0$ and the gauge group $S U(8)$ coupled to an exotic hypermultiplet in the "box" representation, which was introduced in Ref. [23]. This theory cannot be realized in $\mathrm{F}$ theory. The $6 \mathrm{D}$ anomaly cancellation sets the vectors as $a=-3$ and $b=8$.

The 2D SCFTs on a string with charge $Q>0$ in this theory satisfy the conditions (25) and (26). The strongest constraint on the left-moving central charge is given by the minimal string with $Q=1$. The central charge constraint for this model is marginally satisfied as

$$
\frac{k \times 63}{k+8} \leq c_{L} \rightarrow 31.5 \leq 32 \text { for } k=Q \cdot b=8 .
$$

Therefore, at least as far as the unitarity constraint is concerned, this theory is not ruled out, and the strings can consistently couple to this $6 \mathrm{D}$ supergravity theory.

\section{CONCLUSIONS}

In summary, we have discussed the consistencies of 10D and $6 \mathrm{D} \mathcal{N}=(1,0)$ supergravity theories as seen from $2 \mathrm{D}$ 
strings that couple to the 2-forms in the bulk. We have identified the central charges of the world sheet SCFTs on the strings using the anomaly inflow from the bulk supergravity theory. The unitarity of the world sheet SCFTs associated to the central charges leads to novel constraints on the allowed supergravity models, which are not visible from the particle viewpoint.

In this paper, we analyzed only a handful of 6D supergravity models. A large class of would-be consistent $6 \mathrm{D}$ supergravity theories has been discussed in the literature, for example, Refs. [8,23,40]. It might be possible to similarly rule out many such models using more detailed constraints from string probes that we considered in this paper. We leave this for future work.

It would be straightforward to generalize the anomaly inflow consideration discussed in this paper to another type of branes coupled to the supergravity theories. Our discussion in this paper is merely a starting point of a bigger program to understand the consistency of quantum gravitational theories in various dimensions by coupling them to all possible branes and defects of the theories. We hope this program ultimately provides a complete classification of consistent supergravity theories in six and perhaps also other dimensions and more broadly deepens our understanding of the swampland criteria.

\section{ACKNOWLEDGMENTS}

We would like to thank E. Bergshoeff, M. Roček, and W. Taylor for useful and informative discussions. H. K. and G. S. would like to thank Harvard University for hospitality during part of this work. H. K. is supported by the POSCO Science Fellowship of POSCO TJ Park Foundation and the National Research Foundation of Korea Grant No. 2018R1D1A1B07042934. G. S. is supported in part by the DOE Grant No. DE-SC0017647 and the Kellett Award of the University of Wisconsin. The research of C. V. is supported in part by the NSF Grant No. PHY1719924 and by a grant from the Simons Foundation (Grant No. 602883, C. V.).

\section{APPENDIX A: ANOMALIES IN 10D SUPERGRAVITY THEORIES}

We adopt the normalization used in Ref. [42], but a factor of $1 / 4 \pi$ is included in the curvature 2-form $R$, and the field strength $F$ includes a factor of $1 / 2 \pi$. An $\mathcal{N}=1$ supergravity theory in ten dimensions contains a Majorana-Weyl gravitino, some spin- $\frac{1}{2}$ fermions with negative chirality, and gauginos with positive chirality. The gravitino contributes to the anomaly as

$$
I_{12}^{3 / 2}=-\frac{11}{126} \operatorname{tr} R^{6}+\frac{5}{96} \operatorname{tr} R^{4} \operatorname{tr} R^{2}-\frac{7}{1152}\left(\operatorname{tr} R^{2}\right)^{3},
$$

while the contribution from a spin- $\frac{1}{2}$ fermion is

$$
\begin{aligned}
I_{12}^{1 / 2}= & \left(\operatorname{tr}_{\mathcal{R}} 1\right)\left[\frac{1}{5670} \operatorname{tr} R^{6}+\frac{1}{4320} \operatorname{tr} R^{4} \operatorname{tr} R^{2}+\frac{1}{10368}\left(\operatorname{tr} R^{2}\right)^{3}\right] \\
& -\frac{1}{2} \operatorname{tr}_{\mathcal{R}} F^{2}\left[\frac{1}{360} \operatorname{tr} R^{4}+\frac{1}{288}\left(\operatorname{tr} R^{2}\right)^{2}\right] \\
& +\frac{1}{288}\left(\operatorname{tr}_{\mathcal{R}} F^{4}\right) \operatorname{tr} R^{2}-\frac{1}{720} \operatorname{tr}_{\mathcal{R}} F^{6},
\end{aligned}
$$

where $\mathcal{R}$ denotes the representation of the fermion under the gauge algebra. The total one-loop anomaly of the theory is given by the sum over all fermion contributions as

$$
\begin{aligned}
I_{12}^{1-\text { loop }}= & I_{12}^{3 / 2}-\left.I_{12}^{1 / 2}\right|_{\mathcal{R}=1}+\left.I_{12}^{1 / 2}\right|_{\mathcal{R}=a d j} \\
= & \frac{\operatorname{dim} G-496}{5670} \operatorname{tr} R^{6}+\frac{\operatorname{dim} G+224}{4320} \operatorname{tr} R^{4} \operatorname{tr} R^{2} \\
& +\frac{\operatorname{dim} G-64}{10368}\left(\operatorname{tr} R^{2}\right)^{3} \\
& -\frac{1}{2} \operatorname{tr}_{\text {adj }} F^{2}\left[\frac{1}{360} \operatorname{tr} R^{4}+\frac{1}{288}\left(\operatorname{tr} R^{2}\right)^{2}\right] \\
& +\frac{1}{288} \operatorname{tr}_{\text {adj }} F^{4} \operatorname{tr} R^{2}-\frac{1}{720} \operatorname{tr}_{\text {adj }} F^{6},
\end{aligned}
$$

where $\operatorname{dim} G$ is the dimension of the gauge group. When this one-loop anomaly factorizes as

$$
I_{12}^{1-\text { loop }}=X_{4} \wedge X_{8}
$$

it can be canceled by the Green-Schwarz mechanism [12]. This factorization condition allows only four choices of gauge groups: $S O(32), E_{8} \times E_{8}, E_{8} \times U(1)^{248}$, and $U(1)^{496}$.

To cancel the one-loop anomaly, we add to the action the Green-Schwarz term

$$
S^{\mathrm{GS}}=\int B_{2} \wedge X_{8}
$$

Here, the 2-form field $B_{2}$ in the 10D theory transforms under the local gauge and Lorentz group as

$$
B_{2} \rightarrow B_{2}-\frac{1}{4} \operatorname{Tr}(\Lambda F)+\operatorname{tr}(\Theta R),
$$

where $\Lambda$ and $\Theta$ are the transformation parameters. It then follows that the Green-Schwarz term induces anomalies under the gauge and Lorentz transformations, which may cancel the one-loop anomalies. We normalize "Tr" such that the integral of $\frac{1}{4} \operatorname{Tr} F^{2}$ over a 4-manifold gives the instanton number $Q \in \mathbb{Z}$. Note that the gauge transformation of $B_{2}$ is fixed by supersymmetry and the gauge invariance of the 3-form field strength $H_{3}$ [16,17]. The Lorentz transformation of $B_{2}$ is, on the other hand, fixed by the higher-order correction on $\mathrm{H}_{3}$ in the derivative expansion. 


\section{APPENDIX B: ANOMALY INFLOWS FROM SIX TO TWO DIMENSIONS}

Let us briefly review the anomaly inflow computation in 6D theories in the presence of 2D strings discussed in Refs. [28,29] (see also Refs. [43,44] for the anomaly inflow of self-dual strings in the $6 \mathrm{D} \mathcal{N}=(2,0)$ SCFTs). When $Q_{i}$ strings are located at $x^{1,2,3,4}=0$, the Bianchi identity for the 2 -form fields is modified as

$$
d H^{\alpha}=X_{4}^{\alpha}+Q^{\alpha} \prod_{a=1}^{4} \delta\left(x^{a}\right) d x^{a} .
$$

The shift in the right-hand side in the Bianchi identity applies to for the anomaly contribution from the GreenSchwarz term as

$$
\begin{aligned}
I_{8}^{\mathrm{GS}}= & -\frac{1}{2} \Omega_{\alpha \beta}\left(X_{4}^{\alpha}+Q^{\alpha} \prod_{a=1}^{4} \delta\left(y^{a}\right) d y^{a}\right) \\
& \times\left(X_{4}^{\beta}+Q^{\alpha} \prod_{a=1}^{4} \delta\left(y^{a}\right) d y^{a}\right) .
\end{aligned}
$$

As a result, a nontrivial anomaly inflow is induced toward the string world sheet. The anomaly inflow can be computed by integrating the 8-form anomaly polynomial over the four transverse directions to the strings. One computes

$$
I_{4}^{\text {inflow }}=-\Omega_{\alpha \beta} Q^{\alpha}\left(X_{4}^{\alpha}+\frac{1}{2} Q^{\beta} \chi_{4}\left(N_{4}\right)\right) .
$$

This inflow must be canceled by the anomalies arising from the world sheet d.o.f. on the 2D strings. Hence, the anomaly polynomial of the $2 \mathrm{D}$ world sheet SCFT must be

$$
I_{4}=-I_{4}^{\text {inflow }}=\Omega_{\alpha \beta} Q^{\alpha}\left(X_{4}^{\alpha}+\frac{1}{2} Q^{\beta} \chi_{4}\left(N_{4}\right)\right) .
$$

Here, $\chi_{4}\left(N_{4}\right)$ is the Euler class of the $S O(4)=S U(2)_{l} \times$ $S U(2)_{R}$ normal bundle for the transverse $\mathbb{R}^{4}$ directions, and it can also be written as $\chi_{4}\left(N_{4}\right)=c_{2}(l)-c_{2}(R)$ in terms of the second Chern classes $c_{2}(l)$ and $c_{2}(R)$ for $S U(2)_{l}$ and $S U(2)_{R}$.
[1] T. D. Brennan, F. Carta, and C. Vafa, Proc. Sci., TASI2017 (2017) 015.

[2] E. Palti, Fortschr. Phys. 67, 1900037 (2019).

[3] T. Banks, N. Seiberg, and E. Silverstein, Phys. Lett. B 401, 30 (1997)

[4] W. Taylor, arXiv:1104.2051.

[5] M. B. Green, J. H. Schwarz, and E. Witten, Superstring Theory. Vol. 2: Loop Amplitudes, Anomalies and Phenomenology (Cambridge University Press, Cambridge, England, 1988).

[6] C. Vafa, arXiv:hep-th/0509212.

[7] A. Adams, O. DeWolfe, and W. Taylor, Phys. Rev. Lett. 105, 071601 (2010).

[8] V. Kumar, D. R. Morrison, and W. Taylor, J. High Energy Phys. 11 (2010) 118.

[9] J. Polchinski, Int. J. Mod. Phys. A 19, 145 (2004).

[10] T. Banks and N. Seiberg, Phys. Rev. D 83, 084019 (2011).

[11] D. Harlow and H. Ooguri, arXiv:1810.05338.

[12] M. B. Green and J. H. Schwarz, Phys. Lett. 149B, 117 (1984).

[13] C. G. Callan, Jr. and J. A. Harvey, Nucl. Phys. B250, 427 (1985).

[14] J. D. Blum and J. A. Harvey, Nucl. Phys. B416, 119 (1994).

[15] D. Freed, J. A. Harvey, R. Minasian, and G. W. Moore, Adv. Theor. Math. Phys. 2, 601 (1998).

[16] E. Bergshoeff, M. de Roo, B. de Wit, and P. van Nieuwenhuizen, Nucl. Phys. B195, 97 (1982).
[17] G. F. Chapline and N. S. Manton, Phys. Lett. 120B, 105 (1983).

[18] P. Di Francesco, P. Mathieu, and D. Senechal, in Conformal Field Theory, Graduate Texts in Contemporary Physics (Springer-Verlag, New York, 1997).

[19] A. Sagnotti, Phys. Lett. B 294, 196 (1992).

[20] J. H. Schwarz, Phys. Lett. B 371, 223 (1996).

[21] V. Kumar and W. Taylor, Adv. Theor. Math. Phys. 15, 325 (2011).

[22] V. Kumar and W. Taylor, J. High Energy Phys. 12 (2009) 050

[23] V. Kumar, D. S. Park, and W. Taylor, J. High Energy Phys. 04 (2011) 080.

[24] S. B. Johnson and W. Taylor, Fortschr. Phys. 64, 581 (2016).

[25] W. Taylor and A. P. Turner, J. High Energy Phys. 06 (2018) 010.

[26] N. Raghuram and W. Taylor, J. High Energy Phys. 10 (2018) 182.

[27] W. Taylor and A. P. Turner, J. High Energy Phys. 05 (2019) 081

[28] H.-C. Kim, S. Kim, and J. Park, arXiv:1608.03919.

[29] H. Shimizu and Y. Tachikawa, J. High Energy Phys. 11 (2016) 165.

[30] H. Hayashi, P. Jefferson, H.-C. Kim, K. Ohmori, and C. Vafa, arXiv:1905.00116.

[31] B. Haghighat, S. Murthy, C. Vafa, and S. Vandoren, J. High Energy Phys. 01 (2016) 009.

[32] The coefficients of the Gauss-Bonnet term, the Riemannsquared term, and the Weyl-squared term are all equal. This 
can be seen using the equations of motion to rewrite the operators involving $R$ and $R_{\mu \nu}$.

[33] C. Cheung and G. N. Remmen, Phys. Rev. Lett. 118, 051601 (2017).

[34] Y. Hamada, T. Noumi, and G. Shiu, Phys. Rev. Lett. 123, 051601 (2019).

[35] This last condition in F-theory setup translates to the condition that $J \cdot K<0$, signifying that the base of F-theory compactification is positively curved, which is necessary for solving Einstein's equation when $\tau$ varies over the base.

[36] A. Strominger and C. Vafa, Phys. Lett. B 379, 99 (1996).
[37] C. Vafa, Adv. Theor. Math. Phys. 2, 207 (1998).

[38] A. Dabholkar and J. Park, Nucl. Phys. B472, 207 (1996).

[39] A. Dabholkar and J. Park, Nucl. Phys. B477, 701 (1996).

[40] V. Kumar, D. R. Morrison, and W. Taylor, J. High Energy Phys. 02 (2010) 099.

[41] N. Seiberg and E. Witten, Nucl. Phys. B471, 121 (1996).

[42] A. Bilal, arXiv:0802.0634.

[43] M. Henningson, Commun. Math. Phys. 257, 291 (2005).

[44] D. S. Berman and J. A. Harvey, J. High Energy Phys. 11 (2004) 015. 